\title{
Evolutionary conservation of lampbrush-like loops in drosophilids Roberto Piergentili
}

\author{
Address: Dipartimento di Genetica e Biologia Molecolare, "Sapienza" Università di Roma, Piazzale Aldo Moro 5, 00185 Rome, Italy \\ Email: Roberto Piergentili - roberto.piergentili@uniroma1.it
}

Published: 14 August 2007

BMC Cell Biology 2007, 8:35 doi:10.1 |86//47|-2/21-8-35

This article is available from: http://www.biomedcentral.com/I47I-2/2I/8/35

(c) 2007 Piergentili; licensee BioMed Central Ltd.

This is an Open Access article distributed under the terms of the Creative Commons Attribution License (http://creativecommons.org/licenses/by/2.0), which permits unrestricted use, distribution, and reproduction in any medium, provided the original work is properly cited.
Received: II January 2007

Accepted: 14 August 2007

\begin{abstract}
Background: Loopin-I is an abundant, male germ line specific protein of Drosophila melanogaster. The polyclonal antibody T53-FI specifically recognizes Loopin-I and enables its visualization on the Y-chromosome lampbrush-like loop named kl-3 during primary spermatocyte development, as well as on sperm tails. In order to test lampbrush-like loop evolutionary conservation, extensive phasecontrast microscopy and immunostaining with T53-FI antibody was performed in other drosophilids scattered along their genealogical tree.

Results: In the male germ line of all species tested there are cells showing giant nuclei and intranuclear structures similar to those of Drosophila melanogaster primary spermatocytes. Moreover, the antibody T53-FI recognizes intranuclear structures in primary spermatocytes of all drosophilids analyzed. Interestingly, the extent and conformation of the staining pattern is speciesspecific. In addition, the intense staining of sperm tails in all species suggests that the terminal localization of Loopin-I and its orthologues is conserved. A comparison of these cytological data and the data coming from the literature about sperm length, amount of sperm tail entering the egg during fertilization, shape and extent of both loops and primary spermatocyte nuclei, seems to exclude direct relationships among these parameters.

Conclusion: Taken together, the data reported strongly suggest that lampbrush-like loops are a conserved feature of primary spermatocyte nuclei in many, if not all, drosophilids. Moreover, the conserved pattern of the T53-FI immunostaining indicates that a Loopin-I-like protein is present in all the species analyzed, whose localization on lampbrush-like loops and sperm tails during spermatogenesis is evolutionary conserved.
\end{abstract}

\section{Background}

A synthetic description of Drosophila melanogaster spermatogenesis comes from light microscopy studies [1-3], as well as electron microscopy studies [4-9]. At the tip of Drosophila testis a group of 8-9 staminal cells mitotically divide forming another staminal cell and a primary spermatogonium. Primary spermatogonia divide four times producing, after the last division, sixteen primary spermatocytes. Primary spermatocytes undergo a relatively long ( 90 hours) maturation phase, during which they slowly increase their nuclear volume so that by the end of the growth phase they are 25-30 times larger than spermatogonia. Morphologically, at the beginning of their development primary spermatocytes are very similar to spermatogonia; however, the completely developed nuclei are characterized, in addition to their size, by the presence of three filamentous structures called lampbrushlike loops. Primary spermatocytes at late meiotic prophase I show an extensive fragmentation of these loops $[10,11]$. Meiosis produces 64 haploid spermatids which are easily 
recognizable by the association of a phase lucent nucleus with a phase dense mitochondrial derivative (nebenkern) of the same size (approximately $7 \mu \mathrm{m}$ ). In the last phase of spermatogenesis, spermatid nuclei reduce in volume by 200 times as a byproduct of DNA condensation and lose cytoplasmic organelles, while the nebenkern elongates and divides into two symmetric halves between which the sperm axoneme is formed. After sperm tail formation, spermatozoa transfer into seminal vesicles and they are ready to be inseminated into the female. Mature spermatozoa in drosophilids are characterized by very long sperm tails: their size is approximately $1.8 \mathrm{~mm}$ in $D$. melanogaster, $23 \mathrm{~mm}$ in $D$. hydei and almost $60 \mathrm{~mm}$ in $D$. bifurca $[5,12-14]$.

The Y chromosome of D. melanogaster is a submetacentric, completely heterochromatic element representing $12 \%$ of the male genome [15]. The main genetic function of the $\mathrm{Y}$ chromosome is male fertility: $\mathrm{X} / 0$ flies are phenotypically normal males, but they are completely sterile [16]. It has been demonstrated that Y-associated fertility factors play a role only in the male germ line [17] and more specifically inside primary spermatocytes [5]. Three fertility factors (namely $k l-5, k l-3$ and $k s-1$ ) have huge physical dimensions [18], showing a DNA content of $\sim 4,000 \mathrm{~Kb}$ each, this being 100 times longer than an average eukaryotic gene. These uncommon sizes might be partly explained by the fact that they form, inside primary spermatocyte nuclei, three giant lampbrush-like loops [10], and indeed one of the most striking features of Y-loops is their DNA content. Most of the Y chromosome DNA is represented by transposable elements [19] and simple sequence satellite DNA [20-22]. Furthermore, some of these satellites are abundantly transcribed in the ks- 1 and kl-5 loops, but the corresponding transcripts do not migrate into the cytoplasm, and disintegrate together with the loops during meiotic prophase I [23]. The unusual behavior of these transcripts has lead to a debate regarding the functional role of Y-loops in drosophilids. It has been proposed that Y-loops are merely the cytological manifestation of unusually long genes (see [24] for review). Goldstein and coworkers [25] found that mutants lacking $k l-3$ and $k l-5$ loci in D. melanogaster do not express some high molecular weight polypeptides and lack the external dynein arms in the sperm axonemes, suggesting that these regions indeed harbor the structural genes for these proteins. In support of this hypothesis Gepner and Hays [26] found an open reading frame (ORF) coding for a dynein heavy chain inside the $k l-5$ locus (Dhc-Yh3); this gene is conserved also in Drosophila hydei in both location (Y chromosome) and cytological phenotype (a loop called Thread) [27]. Further support for this hypothesis comes from Carvalho and coworkers [28] who found another dynein heavy chain gene inside the $k l-3$ fertility factor and an occludin-related gene (ORY) inside the $k s-1$ locus [29].
However, although the Y loops appear to represent single giant transcription units, the presence of such extremely large introns full of repetitive DNA suggests the non-protein coding regions of the transcription units might serve other purposes. Loops can be selectively stained by Giemsa at pH10, as well as by dansyl-chloride and Coomassie blue [10]; furthermore, several antibodies have been raised which specifically recognize these structures [10,30-37], showing that they accumulate high quantities of proteins. An interesting example is provided by T53-1, a monospecific, polyclonal antibody that recognizes Loopin-1, a testis-specific autosomal protein of Drosophila melanogaster. Loopin-1 is present onto both the Ychromosome kl-3 loop and the sperm tails [30]; notably, T53-1 also stains the Pseudonucleolus and, partially, the Cones Y-loops of D. hydei. The accumulation on the loops of proteins encoded by autosomal genes suggests that the Y-loops, besides any coding function, might also play a structural role, possibly as a nuclear framework useful for docking and/or modifying proteins to be used later in spermatogenesis $[10,30,38]$.

In the present paper I studied the male germ lines of 13 Drosophila species and used the T53-F1 antibody (a second antiserum prepared against Loopin-1 which has the same specificity of the original T53-1 antiserum), to assay the evolutionary conservation of lampbrush-like loops. In all the 13 species of drosophilids analyzed, intranuclear structures were detected in primary spermatocytes using phase-contrast microscopy. Such structures resemble the Y-chromosome loops of D. melanogaster [5] and D. hydei [40], and in each species one loop is intensely stained by the antibody. The antibody shows no specific staining in spermatids but strongly decorates sperm tails. These data strongly suggest that lampbrush-like loops are a conserved, and consequently a necessary feature of drosophilid meiosis. Moreover, the conservation of spatial and temporal immunostaining in all species suggests a conserved, although unknown, function for Loopin-1. Comparison of these data with other spermatogenesis parameters, such as the size of primary spermatocyte nuclei or the sperm length, did not reveal any evident relationship, suggesting that these aspects of male germ line are not directly correlated.

\section{Results \\ Phase-contrast analysis}

In order to study the evolutionary conservation of lampbrush-like loops among drosophilids (see the phylogeny in Figure 1), a detailed cytological analysis of male meiosis was carried out using phase-contrast microscopy in 13 species (Table 1). This analysis was performed using the same protocol described for D. melanogaster [30]. Adult or larval testes were dissected in phosphate buffer, gently squashed and immediately frozen in liquid nitrogen. After 

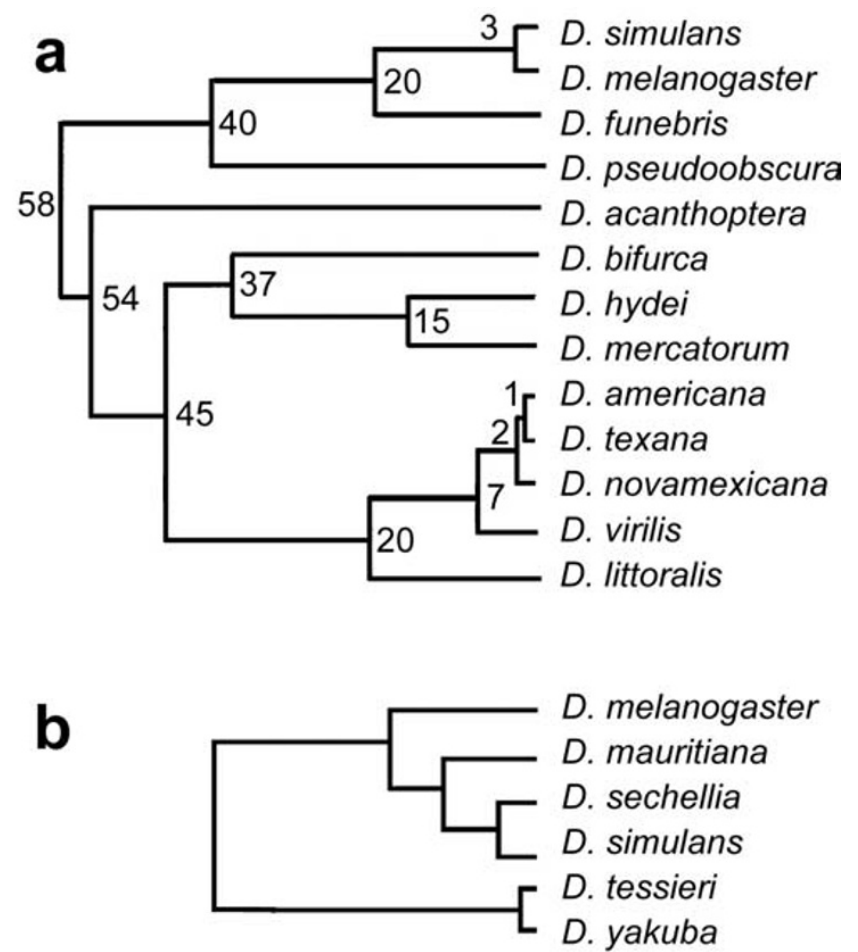

Figure I

Genealogical trees of the drosophilids analyzed in the present work. a: general phylogeny; b: partial phylogeny of the melanogaster subgroup, an enlargement of the simulans/ melanogaster branch of the upper tree. Numbers in panel a indicate how many million years ago the species diverged. The common ancestor of all these species is supposed to have lived some 60 million years ago; the divergence between $D$. melanogaster and $D$. tessieri probably occurred between 10 and 15 million years ago. The upper tree is partially taken and modified from [13]. The lower tree is partially taken and modified from [48].

removal of the coverslip all preparations were fixed using standard methanol/acetone protocol. The results, summarized in Table 1 and illustrated in Figure 2, show that in every species primary spermatocytes could be identified based on their characteristic features, such as their large nuclear size and the presence of prominent intranuclear structures in addition to the nucleolus. Notably, these features are also typical in spermatocytes of the two well characterized species D. melanogaster and D. hydei. In many species (D. acanthoptera, D. bifurca, D. littoralis, D. mauritiana, D. sechellia, D. tessieri, D. yakuba) a spherical structure having a phase dense appearance is visible, which most probably corresponds to the nucleolus as it is similar in both shape and size to D. melanogaster or D. hydei nucleoli. This structure is quite different from most of the other filamentous material visible inside primary spermatocyte nuclei of all species, showing their complex cytological organization before meiosis I. A species-specific pattern for the intranuclear structures is also recognizable. In some cases it is possible to see prominent structures occupying most of the nuclear volume, as for $D$. acanthoptera (Figure 2a), D. americana (Figure 2b), D. funebris (Figure 2d), D. littoralis (Figure 2e), D. sechellia (Figure $2 \mathrm{i}$ ), D. simulans (Figure $2 \mathrm{k}$ ), and D. texana (Figure $2 \mathrm{l}$ ). In other cases these structures are smaller (D. bifurca, Figure $2 \mathrm{c} ; D$. mercatorum, Figure $2 \mathrm{~g} ; D$. yakuba, Figure $2 \mathrm{~m}$ ) or barely visible (D. mauritiana, Figure 2f; D. pseudoobscura, Figure $2 \mathrm{~h}$; D. tessieri, Figure $2 \mathrm{j}$ ). Besides the size, also the shape of these intranuclear structures is species-specific; some of them have a filamentous aspect as seen in D. melanogaster loops, while other species show phase dense structures resembling some of the Y-loops of $D$. hydei (Table 1). Taken together, these data indicate that lampbrush-like structures similar to those already known in $D$. melanogaster and D. hydei are present in all species analyzed. It is therefore likely that these structures are present in all drosophilids, strongly suggesting a conserved role for lampbrush-like loops during spermatogenesis.

\section{Immunostaining by T53-FI}

Following the rationale stated above, testes from the same drosophilid species were fixed and immunostained with the T53-F1 antibody. Although T53-F1 was prepared against a recombinant form of Loopin-1, its specificity is the same as that of the original T53-1 antibody, which was prepared against Loopin-1 purified from Drosophila testes [39]. In all cases the antibody intensely decorates sperm tails (data not shown) and a specific structure inside primary spermatocyte nuclei (Figure 2). Thus a Loopin-1 related protein with a similar function appears to be present in all these species, although this indication was not confirmed by immunoblot analysis. However, clear differences exist among the species. For example in both $D$. funebris (Figure 2d') and D. bifurca (Figure 2c') the T53F1 antibody stains small, dot-like loops, while in D. mercatorum (Figure $2 \mathrm{~g}^{\prime}$ ) it decorates a discoidal structure occupying the central region of the "dumbbell-shaped" primary spermatocyte nuclei. It is noteworthy that the structures recognized by the antibody do not always overlap with structures visible by phase-contrast microscopy. In some species (D. bifurca, D. funebris, D. littoralis, D. mercatorum, D. pseudoobscura) the overlap is evident, in other cases (D. acanthoptera, D. americana, D. mauritiana, D. sechellia, D. simulans, D. texana) it is only partial. There are also species (D. tessieri, D. yakuba) in which there is no overlap. Notwithstanding the morphological differences of the intranuclear structures recognized by T53-F1, these data clearly show that in all the 13 species tested, as well as in the other two species partially described by Pisano and coworkers [30], structures similar to D. melanogaster or D. hydei Y-chromosome lampbrush-like loops are present inside primary spermatocyte nuclei. Moreover, in 


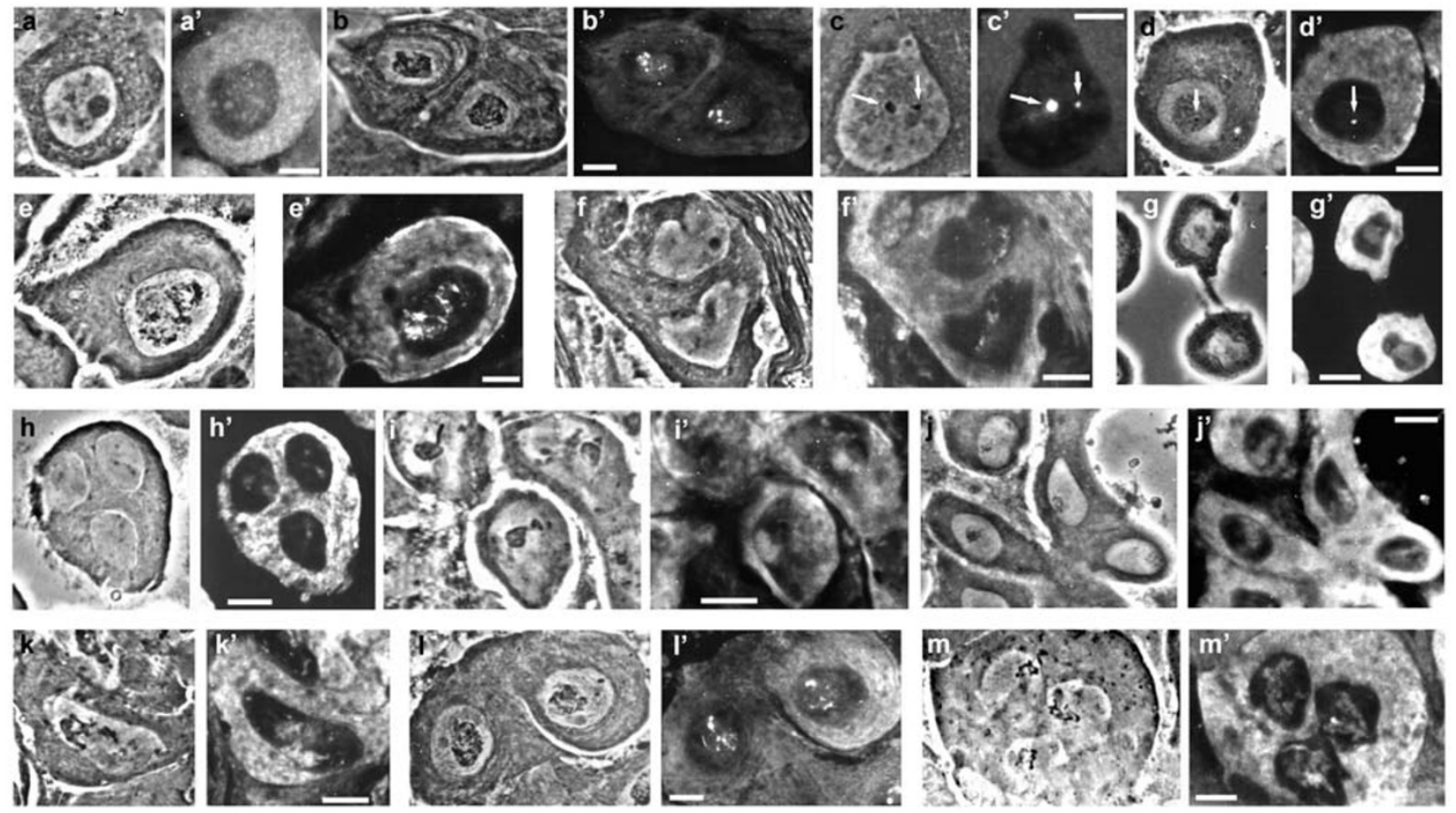

\section{Figure 2}

T53-F I immunostaining of primary spermatocyte nuclei of drosophilids. Note that in all species it is possible to recognize intranuclear structures specifically decorated by the antibody. For all species, the first image is a phase-contrast micrograph, the second shows the corresponding immunostaining. a-a': D. acanthoptera. b-b': D. americana. c-c': D. bifurca. d-d': D. funebris. e-e': D. littoralis. f-f': D. mauritiana. g-g': D. mercatorum. h-h': D. pseudoobscura. i-i': D. sechellia. j-j': D. tessieri. k-k': D. simulans. I-I': D. texana. m-m': D. yakuba. Bars: $10 \mu \mathrm{m}$.

all cases at least one loop is decorated by the T53-F1 antibody.

\section{Evaluation of other cytological parameters}

In order to evaluate possible correlations between the structures revealed by T53-F1 antibody and other cytological parameters of male meiosis, other aspects (both preand post-meiotic) were considered. In particular, data available from the literature about sperm tail length and the amount of sperm tail entering the egg upon fertilization were considered. In addition, data about the shape of primary spermatocyte nuclei obtained in the present work or coming from the literature were collected. These parameters were compared to the relative size and shape of the lampbrush-like loops visible by immunofluorescence. It should be noted however that to date there are no data to assess if the immunostaining completely overlaps the actual size of the loops. Data used for these comparisons are summarized in Table 1 . Alongside the data collected here, those of other four species (D. melanogaster, D. hydei, D. virilis and D. novamexicana) are also reported
[30]. Preliminary results seem to exclude any direct correlation among these parameters.

Drosophilids are known to have sperm tails of extremely variable sizes, starting from $D$. persimilis (only $0.32 \mathrm{~mm}$, similar in size to the $0.36 \mathrm{~mm}$ of the closely related $D$. pseudoobscura analyzed in this paper) to the giant $\sim 60 \mathrm{~mm}$ sperm produced by D. bifurca [14]. Comparison of sperm lengths and relative loop staining extension inside primary spermatocyte nuclei reveals that these two parameters are not correlated. Indeed it is possible to find longtailed species with a small loop (D. bifurca, Figure $\left.2 c-2 c^{\prime}\right)$ as well as species with relatively short sperm tails and a very prominent loop (D. tessieri, Figure $2 \mathrm{j}-2 \mathrm{j}$; D. simulans, Figure $\left.2 \mathrm{k}-2 \mathrm{k}^{\prime}\right)$. Moreover, similarly sized sperm tails of $D$. acanthoptera (Figure 2a-2a'), D. americana (Figure 2b-2b') and D. texana (Figure 2l-2l') do not correspond to similarly sized loops.

The absence of a correlation is also evident when considering the amount of sperm tail entering the egg upon fer- 
Table I: Analysis of various aspects of male spermatogenesis in different drosophilids

\begin{tabular}{|c|c|c|c|c|c|c|c|}
\hline $\begin{array}{l}\text { species (in } \\
\text { alphabetical } \\
\text { order) }\end{array}$ & $\begin{array}{c}\text { sperm length } \\
(\mathrm{mm})\end{array}$ & $\begin{array}{c}\text { sperm tail } \\
\text { entering eggs } \\
(\mathrm{mm})\end{array}$ & $\begin{array}{c}\text { T53-FI } \\
\text { staining in } \\
\text { tails }\end{array}$ & loop size & Loop shape & overlap & nucleus shape \\
\hline D. acanthoptera & $5.83 \pm 0.09$ & $(++)$ & yes & 3 & $d$ & weak & $\mathrm{S}$ \\
\hline D. americana & $5.22 \pm 0.02$ & nd & yes & 1 & $d$ & weak & $\mathrm{S}$ \\
\hline D. bifurca & $58.29 \pm 0.67$ & 1.6 & yes & I & $d$ & strong & $\mathrm{O}$ \\
\hline D. funebris & 8.29 & nd & yes & I & $d$ & strong & $\mathrm{S}$ \\
\hline D. hydei & $23.32 \pm 0.51$ & 1.31 & yes & 2 & $d$ & strong & $\mathrm{O}$ \\
\hline D. littoralis & $7.72 \pm 0.08$ & $(++)$ & yes & 3 & $f$ & strong & $\mathrm{S}$ \\
\hline D. mauritiana & 1.036 & nd & yes & 2 & $f$ & weak & $\mathrm{S}$ \\
\hline D. melanogaster & $1.9 \pm 0.01$ & 1.78 & yes & 3 & $f$ & strong & $\mathrm{S}$ \\
\hline D. mercatorum & nd & nd & yes & 3 & $d$ & strong & 0 \\
\hline D. novamexicana & $6.72 \pm 0.15$ & nd & yes & 3 & $f$ & weak & $\mathrm{S}$ \\
\hline $\begin{array}{l}\text { D. } \\
\text { pseudoobscura }\end{array}$ & 0.36 & 0.36 & yes & 2 & $d$ & strong & $\mathrm{S}$ \\
\hline D. sechellia & 1.649 & nd & yes & 2 & $d$ & weak & $\mathrm{S}$ \\
\hline D. simulans & $\mathrm{I} .14 \pm 0.01$ & I.14 & yes & 3 & $d$ & weak & $\mathrm{S}$ \\
\hline D. tessieri & 1.0 to 2.0 & nd & yes & 3 & $d$ & none & $\mathrm{S}$ \\
\hline D. texana & $5.08 \pm 0.04$ & nd & yes & 2 & $d$ & weak & $\mathrm{S}$ \\
\hline D. virilis & $5.70 \pm 0.16$ & nd & yes & 3 & $f$ & weak & $\mathrm{S}$ \\
\hline D. yakuba & $1.68 \mid$ & nd & yes & 3 & $f$ & none & $S$ \\
\hline
\end{tabular}

Data reported in the first three columns are partially taken from [13,4I, 49-5I], and (++) indicates that almost the entire sperm tail enters the egg. Columns 5, 6 and 7 refer to the structures decorated by T53-FI inside primary spermatocytes, as described in the Results section. In particular, loop size refers to the relative extension of the immunostaining inside the primary spermatocyte nucleus, 3 meaning very extensive loop, and I meaning very small loop. Loop shape indicates its filamentous (f) or dense (d) appearance. Overlap is "strong" when immunostaining and phasecontrast visible structures inside primary spermatocytes nuclei are the same, "weak" when the structures are only partially overlapped; "none" indicates that immunostained structures do not have a counterpart visible by phase-contrast. Nucleus shape, $\mathrm{S}$ indicates spherical or nearly spherical, $O$ indicates other shapes (see Figure 2 and the Discussion section for further explanations). Cytological data about $D$. melanogaster, $D$. hydei, $D$. virilis and D. novamexicana partially come from [30].

tilization and the cytological appearance of loop structures by immunostaining. For example in D. bifurca only a small part of the tail enters the egg, and the loop staining appears small, but in the closely related D. hydei, although only a small part of the tail enters the egg, the loop Pseudonucleolus is much larger. Comments about the utilization by the zygote or by the females of part of sperm tails after fertilization can be found in [41] and references therein.

\section{Discussion}

In the present study an extensive analysis of the male germ line of several Drosophila species was performed, in order to find conserved features. The selected species are scattered along their genealogical tree, so that it was possible to analyze even drosophilids which diverged approximately 58 million years ago (Figure 1a). Cytological analysis was performed using both phase-contrast microscopy and immunofluorescence, and the same fixing procedure was used, so it is possible to argue that the structures stained are probably evolutionary conserved. The results reported here indicate that in all species tested it is possible to recognize cells which are probably mature primary spermatocytes, as both their size and the presence of intranuclear structures are similar to both $D$. melanogaster and
D. hydei (Figure 2). Moreover, with regard to their size and shape, the intranuclear structures are species-specific, and at least one of them is always specifically immunostained by T53-F1, a monospecific polyclonal antibody raised against the Loopin-1 protein of D. melanogaster [39]. In a pattern similar to that seen in D. melanogaster, the staining by T53-F1 is not present in the spermatids of any species, but the antibody intensely stains sperm tails from when they start developing (data not shown). This indicates that a Loopin-1-like protein is loaded onto lampbrush-like loops before meiosis and then accumulates along sperm tails in all the drosophilids analyzed.

Several cytological parameters were considered in order to find possible correlations among them, to elucidate a possible role of Loopin-1, since no mutation is known to date in the loopin-1 gene. However, it was not possible to find any relationship. I found that sperm length, amount of sperm tail inside the egg upon fertilization, loop staining size and loop shape are independent parameters (Table 1 and Figure 2).

Whereas Loopin-1 immunostaining was found to be conserved with regard to its presence in male germ lines, the shape and staining size of the corresponding lampbrush- 
like loops were found to be species-specific. In fact, although all species tested have loops stained by T53-F1 and similarly sized primary spermatocytes nuclei, all possible combinations can be found. The loop appears very small in D. americana, D. bifurca and D. funebris (very distant species) and it appears very prominent in $D$. acanthoptera, D. littoralis, D. melanogaster and D. mercatorum (similarly distant species) (Figure 1). However, it seems that the shape of primary spermatocyte nuclei may be conserved. The only three species having a non spherical nucleus are D. bifurca, D. hydei and D. mercatorum, which are evolutionary close to each other (Figure 1a). The first two show a "pear-like" nucleus, while the last species has an "dumbbell-shaped" nucleus (Figure 2 and Table 1). Notably, all three species have loops of a dense nature, suggesting a similar molecular organization. In this respect it is interesting to note that a filamentous loop recognized by T53-F1 is present only in some species of the melanogaster subgroup (Figure 1B), and in the closely related species $D$. novamexicana, $D$. virilis and $D$. littoralis (Table 1). The conservation of nuclei shape, staining size and appearance is even more interesting if we compare it with previous reports $[42,43]$. The sperm tail length and sperm head size are very variable not only among species, but also among different populations of the same species and even inside the same individual [44]. This suggests that mechanisms controlling premeiotic stages are more stringent than those controlling sperm development.

Thus far the biological role of Loopin-1 in Drosophila melanogaster male germ line is unknown. The loopin-1 gene maps to polytene region 53C [39] and its product was originally described as a tektin-like protein [30]. Subsequent studies, however, demonstrated that the loopin-1 gene corresponds to the CG4750 locus of D. melanogaster and is homologous to a leucine aminopeptidase [39,45]; therefore, it is unrelated to the tektin family of proteins. The protein encoded by this gene is conserved at least in D. simulans (55\% identities, $72 \%$ positives) which diverged from $D$. melanogaster $3 \mathrm{My}$ ago, and in $D$. pseudoobscura (78\% identities, $88 \%$ positives) which diverged from D. melanogaster $40 \mathrm{My}$ ago (Figure 1 ). This is not surprising, since it has been recently demonstrated that leucine aminopeptidases represent an important fraction of the Drosophila sperm proteome [49], although their role in sperm tail is still unclear. It is also known that Loopin-1 localization in D. melanogaster sperm tails is not dependent upon loop binding [30]. Indeed, its pattern of localization is quite peculiar: the protein recognized by the T53F1 antibody appears inside primary spermatocyte nuclei during their maturation, binding to the kl-3 loop throughout its development [30]. At the beginning of meiosis, as soon as Y-chromosome lampbrush-like loops disgregate, staining fades and no other sign of the protein is present during meiosis or spermatid maturation. The staining once again becomes intense in sperm tails at the time they begin to elongate. Notably, this peculiar behavior was found to be similar in all the 13 species examined, despite their considerable phylogenetic distance (Figure 1). The fact that T53-F1 reacts specifically in a conserved pattern strongly suggests that $D$. melanogaster Loopin-1 is a conserved protein and that the formation of loop-like structures during primary spermatocyte development is a necessary step during spermatogenesis in all species examined.

\section{Conclusion}

The data reported here show that a Loopin-1-like protein is detectable in both primary spermatocyte nuclei and sperm tails in all species tested, and possibly in all drosophilids. These data strongly suggest that this protein might have an important functional role during spermatogenesis through its binding to intranuclear structures during the development of primary spermatocyte nuclei. Notably, the structures decorated by the T53-F1 antibody during primary spermatocyte nuclei development clearly resemble the well known Y-chromosome lampbrush-like loops of D. melanogaster and $D$. hydei. It is likely that lampbrush-like loops might be a sign of Y chromosome activity during spermatogenesis in all drosophilids tested. Therefore it would not be surprising to find that the Y chromosome plays a similar role in the development of lampbrush-like loops in all drosophilids.

Evolutionary data demonstrate that drosophilids diverged at least 60 million years ago. Data shown here support the hypothesis that a Loopin-1-like protein exists in all species tested, indicating that it is evolutionary conserved. Moreover, data presented here also demonstrate that primary spermatocyte nuclei of all species tested show the presence of discrete intranuclear structures, indicating that this cytological feature is also conserved. The lack of a loopin-1 mutation however has impeded the assessment of its role during spermatogenesis, and the function of Loopin-1 loading onto lampbrush-like loops in the male germ line. Interestingly, mutations in other genes that, like loopin-1, are located on the autosomes have recently been shown to affect the formation of the kl-3 loop and to alter the external dynein arms of the sperm axonemes [47] which are believed to be the site of action of the dynein encoded by the $k l-3$ fertility factor. The spermatozoa of these mutants either degenerate before maturation or have major structural defects leading to their complete immobility. These data suggest that these autosomal proteins might be involved in building the structure of the lampbrush-like loops - either to set up structures necessary for transcription of the $k l-3$ gene or to perform other functions associated with the loop. 


\section{Methods \\ Strains}

D. bifurca, D. acanthoptera and D. littoralis were kindly provided by the National Drosophila Species Resource Center at Bowling Green State University ( $\mathrm{OH}$ - USA); all the other species are part of the collection of Drosophila stocks of Prof. M. Gatti and Prof. S. Bonaccorsi at the University of Rome "Sapienza". All lines were reared at $25^{\circ} \mathrm{C}$ using standard cornmeal medium.

\section{Cytology and immunostaining}

Larvae or adults of all species were dissected and testes were fixed and analyzed as described in [30], with the exception of very long testes, as in D. bifurca. In this case $24 \times 32 \mathrm{~mm}$ coverslips were used, and testes were gently unfolded with forceps before squashing. This protocol preserves the intranuclear structures visible during in vivo analysis, as demonstrated for D. melanogaster and D. hydei. The T53-F1 antibody was kindly provided by Dr. Pisano. The T53-1 antibody was obtained injecting Drosophila melanogaster testis proteinaceous extracts into mice. Subsequently the loopin-1 gene was identified (CG4750) using the T53-1 antibody to isolate E. coli clones expressing the protein. This recombinant protein was again injected into mice and the T53-F1 antibody was obtained. The complete procedure for the isolation of bacterial clones and the characterization of the T53-F1 staining pattern by both immunofluorescence and western blot analyses were extensively described in the PhD Thesis of Dr. Gambino [39]. Since there is no exhaustive description of male germ lines of the species used in the present work, it was assumed that the largest cells observed inside testes, which also showed intranuclear structures besides the nucleolus, were indeed mature primary spermatocytes, as is the case for D. melanogaster and D. hydei.

\section{Authors' contributions}

$\mathrm{RP}$ designed the study, carried out all experiments and prepared the manuscript.

\section{Acknowledgements}

I would like to thank the National Drosophila Species Resource Center at Bowling Green State University (OH - USA) for the species D. bifurca, D. acanthoptera and D. littoralis, and Prof. M. Gatti and Prof. S. Bonaccorsi ("Sapienza" Università di Roma - Italy) for the other species used during this work. I am also grateful to Dr. C. Pisano for the T53-FI antibody. This work was not supported by any grant, for this I am deeply indebted with Prof. M. Gatti for all logistic support. Finally, I am grateful to Prof. S. Pitnick (Syracuse University, NY - USA), Prof. P. Dimitri, Prof. S. Bonaccorsi and Dr. A. Wainman ("Sapienza" Università di Roma - Italy), for critical reading of the manuscript.

\section{References}

I. Cooper KW: Normal spermiogenesis in Drosophila. In Biology of Drosophila Edited by: Demerec M. New York and London, Hafner Publishing Company; 1965:I-6I.
2. Kemphues KJ, Kaufman TC, Raff RA, Raff EC: The testis-specific $\beta$ tubulin subunit in Drosophila melanogaster has multiple functions in spermatogenesis. Cell 1982, 31:655-670.

3. Fuller MT: Genetic analysis of spermatogenesis in Drosophila: the role of the testis-specific $\beta$-tubulin and interacting genes in cellular morphogenesis. In Gametogenesis and the Early Embryo Edited by: Gall JG. New York, Alan R. Liss; 1986:19-42.

4. Tates AD: Cytodifferentiation during spermatogenesis in Drosophila melanogaster. PhD Thesis I97I [http://flybase.bio.indi ana.edu/reports/FBrf0023l03.html]. Leiden University

5. Lindsley DL, Tokuyasu KT: Spermatogenesis. In The Genetics and Biology of Drosophila Volume 2. Edited by: Ashburner M, Wright TRF. New York, Academic Press; 1980:225-294.

6. Lin HPP, Ault JG, Church K: Meiosis in Drosophila melanogaster. I. Chromosome identification and kinetochore microtubule numbers during the first and second meiotic divisions in males. Chromosoma 1981, 83:507-521.

7. Church K, Lin HPP: Meiosis in Drosophila melanogaster: the prometaphase-I kinetochore microtubule bundle and kinetochore orientation in males. J Cell Biol 1982, 93:365-373.

8. Church K, Lin HPP: Kinetochore microtubules and chromosome movement during prometaphase in Drosophila melanogaster spermatocytes studied in life and with the electron microscope. Chromosoma 1985, 92:273-282.

9. Nielsen MG, Raff EC: The best of all worlds or the best possible world? Developmental constraint in the evolution of $\beta$-tubulin and the sperm tail axoneme. Evol Dev 2002, 4:303-3I5.

10. Bonaccorsi S, Pisano C, Puoti F, Gatti M: Y chromosome loops in Drosophila melanogaster. Genetics 1988, 120:1015-1034.

II. Cenci G, Bonaccorsi S, Pisano C, Vernì F, Gatti M: Chromatin and microtubule organization during premeiotic, meiotic and early postmeiotic stages of Drosophila melanogaster spermatogenesis. J Cell Sci 1994, 107:3521-3534.

12. Lifschytz $\mathrm{E}$ : The genetic control of spermatogenesis. Int Rev Cytol 1987, 109:21I-258.

13. Pitnick S, Markow TA, Spicer GS: Delayed male maturity is a cost of producing large sperm in Drosophila. Proc Natl Acad Sci USA 1995, 92:10614-10618.

14. Pitnick S, Spicer GS, Markow TA: How long is a giant sperm? Nature 1995, 375:109.

15. Pimpinelli S, Santini G, Gatti M: Characterization of Drosophila heterochromatin. II. C- and N-banding. Chromosoma 1976, 57:377-386.

16. Bridges CB: Non-disjunction as a proof of the chromosome theory of heredity. Genetics 1916, I:1-52. 107-163

17. Marsh JL, Wieschaus E: Is sex determination in germ line and soma controlled by separate genetic mechanisms? Nature 1978, 272:249-251.

18. Gatti M, Pimpinelli S: Cytological and genetic analysis of the $\mathbf{Y}$ chromosome of Drosophila melanogaster. I. Organization of the fertility factors. Chromosoma 1983, 88:349-373.

19. Pimpinelli S, Berloco M, Fanti L, Dimitri P, Bonaccorsi S, Marchetti E, Caizzi R, Caggese C, Gatti M: Transposable elements are stable structural components of Drosophila melanogaster heterochromatin. Proc Natl Acad Sci USA 1995, 92:3804-3808.

20. Peacock WJ, Lohe AR, Gerlach WL, Dunsmuir P, Dennis ES, Appels $R$ : Fine structure and evolution of DNA in heterochromatin. Cold Spring Harbor Symp Quant Biol 1978, 42: I I 2 I-1 I 35.

21. Brutlag DL: Molecular arrangement and evolution of heterocromatic DNA. Annu Rev Genet 1980, I4:121-I44.

22. Bonaccorsi S, Lohe A: Fine mapping of satellite DNA sequences along the $Y$ chromosome of Drosophila melanogaster: relationships between satellite sequences and fertility factors. Genetics 1991, I29:177-189.

23. Bonaccorsi S, Gatti M, Pisano C, Lohe A: Transcription of satellite DNA on the $Y$ chromosome loops of Drosophila melanogaster. Chromosoma 1990, 99:260-266.

24. Hackstein JHP, Hochstenbach R: The elusive fertility genes of Drosophila: the ultimate haven for selfish genetic elements. Trends Genet 1995, I I:195-200.

25. Goldstein LSB, Hardy RW, Lindsley DL: Structural genes on the $\mathbf{Y}$ chromosome of Drosophila melanogaster. Proc Natl Acad Sci USA 1982, 79:7405-7409.

26. Gepner J, Hays TS: $\mathbf{A}$ fertility region on the $\mathbf{Y}$ chromosome of Drosophila melanogaster encodes a dynein microtubule motor. Proc Natl Acad Sci USA 1993, 90: I I I32-I I I 36. 
27. Kurek R, Reugels AM, Glätzer KH, Bünemann H: The $\mathbf{Y}$ chromosomal fertility factor Threads in Drosophila hydei harbors a functional gene encoding an axonemal dynein $\beta$ heavy chain protein. Genetics 1998, | 49:|363-1376.

28. Carvalho AB, Lazzaro BP, Clark AG: Y chromosomal fertility factors $\mathbf{k l - 2}$ and $\mathbf{k l - 3}$ of Drosophila melanogaster encode dynein heavy chain polypeptides. Proc Natl Acad Sci USA 2000, 97:13239-13244.

29. Carvalho AB, Dobo BA, Vibranovski MD, Clark AG: Identification of five new genes on the $\mathbf{Y}$ chromosome of Drosophila melanogaster. Proc Natl Acad Sci USA 200 I, 98: I 3225-13230.

30. Pisano C, Bonaccorsi S, Gatti M: The kl-3 loop of the Y chromosome of Drosophila melanogaster binds a tektin-like protein. Genetics 1993, 133:569-579.

31. Saumweber H, Symmons P, Kabish R, Will H, Bonhoeffer F: Monoclonal antibodies against chromosomal proteins of Drosophila melanogaster. Chromosoma 1980, 80:253-275.

32. Risau W, Symmons P, Saumweber H, Frash M: Non packaging and packaging proteins of hnRNA in Drosophila melanogaster. Cell 1983, 33:529-54|.

33. Menzel S, Glatzer KH: Localization of RNP antigens in primary spermatocytes of Drosophila melanogaster by indirect immunofluorescence and their correlation to fertility factors. Dros Info Serv 1985, 61:121 [http://flybase.bio.indiana.edu/ reports/FBrf0042219.html].

34. Eberhart CG, Maines JZ, Wasserman SA: Meiotic cell cycle requirement for a fly homologue of human Deleted in Azoospermia. Nature 1996, 38 1:783-785.

35. Heatwole VM, Haynes SR: Association of RB97D, an RRM protein required for male fertility, with a $Y$ chromosome lampbrush loop in Drosophila spermatocytes. Chromosoma 1996 105:285-292.

36. Lu AQ, Beckingham K: Androcam, a Drosophila calmodulinrelated protein, is expressed specifically in the testis and decorates loop kl-3 of the $\mathbf{Y}$ chromosome. Mech Dev 2000, 94:17|-|8|.

37. Frank DJ, Martin SR, Gruender BN, Lee YS, Simonette RA, Bayley PM, Miller KG, Beckingham KM: Androcam is a tissue-specific lightchain for myosin VI in the Drosophila testis. J Biol Chem 2006, 28 I:24728-24736.

38. Hennig W, Brand RC, Hackstein JHP, Hochstenbach R, Kremer H Lankenau DH, Lankenau S, Miedema K, Potgens A: Y chromosomal fertility genes of Drosophila: a new type of eukaryotic genes. Genome 1989, 3 1:561-571.

39. Gambino I: Clonaggio e analisi di tekl-3, un gene che codifica una proteina associata alla loop $\mathbf{k l - 3}$ del cromosoma $\mathbf{Y}$ d Drosophila melanogaster. PhD Thesis 1993 [http://flybase.bio.ind ana.edu/reports/FBrfO |667I I.html]. Università di Roma "La Sapienza"

40. Hennig W, Kremer H: Spermatogenesis of Drosophila hydei. Int Rev Cytol 1990, I 23: I29-I75.

4I. Pitnick S, Markow TA: Male gametic strategies: sperm size, testes size, and the allocation of ejaculate among successive mates by the sperm-limited fly Drosophila pachea and its relatives. Am Nat 1994, 143:785-819.

42. Stevenson RD, Hill MF, Bryant PJ: Organ and cell allometry in Hawaiian Drosophila: how to make a big fly. Proc Biol Sci 1995 , 259: 105-II0.

43. McNeill Alexander R: Big flies have bigger cells. Nature 1995 , 375:20.

44. Joly D, Korol A, Nevo E: Sperm size evolution in Drosophila: inter- and intraspecific analysis. Genetica 2004, 1 20:233-244.

45. Gatti M: Genetic and molecular organization of the $\mathbf{Y}$ chromosome fertility factors of Drosophila melanogaster. Scient Rept Inst Pasteur, Roma 199/-1994 1995:25-26 [http://flybase.bio.indi ana.edu/reports/FBrf008202I.html].

46. Dorus S, Busby SA, Gerike U, Shabanowitz J, Hunt DF, Karr TL: Genomic and functional evolution of the Drosophila melanogaster sperm proteome. Nat Genet 2006, 38: I440-I 445

47. Piergentili R, Bonaccorsi S, Raffa GD, Pisano C, Hackstein JHP, Mencarelli C: Autosomal control of the Y-chromosome kl-3 loop of Drosophila melanogaster. Chromosoma 2004, I I 3:188-196.

48. Lewis RL, Beckenbach AT, Mooers AO: The phylogeny of the subgroups within the melanogaster species group: likelihood tests on COI and COII sequences and a Bayesian estimate of phylogeny. Mol Phylogenet Evol 2005, 37:15-24.
49. Bressac C, Joly D, Devaux J, Lachaise D: Can we predict the mating pattern of Drosophila females from the sperm length distribution in males? Experientia 1991, 47: III-II4.

50. Joly D, Bressac C, Devaux J, Lachaise D: Sperm lenght diversity in Drosophilidae. Dros Inf Serv 1991, 70:104-108 [http://fly base.bio.indiana.edu/reports/FBrf0053516.html]

51. Karr TL, Pitnick S: The ins and outs of fertilization. Nature 1996 , 379:405-406
Publish with Bio Med Central and every scientist can read your work free of charge

"BioMed Central will be the most significant development for disseminating the results of biomedical research in our lifetime. "

Sir Paul Nurse, Cancer Research UK

Your research papers will be:

- available free of charge to the entire biomedical community

- peer reviewed and published immediately upon acceptance

- cited in PubMed and archived on PubMed Central

- yours - you keep the copyright 